\title{
Wake up and smell the coffee: caffeine supplementation and exercise performance-an umbrella review of 21 published meta-analyses
}

\author{
Jozo Grgic (1) ,' Ivana Grgic, ${ }^{2}$ Craig Pickering (1) , ${ }^{3,4}$ Brad J Schoenfeld, \\ David J Bishop, ${ }^{1,6}$ Zeljko Pedisic
}

- Additional material is

published online only. To view please visit the journal online (http://dx.doi.org/10.1136/ bjsports-2018-100278).

${ }^{1}$ Institute for Health and Sport (IHES), Victoria University, Melbourne, Australia ${ }^{2}$ County Hospital

Schrobenhausen,

Schrobenhausen, Germany ${ }^{3}$ Institute of Coaching and Performance, School of Sport and Wellbeing, University of Central Lancashire, Preston, UK ${ }^{4}$ Exercise and Nutritional Genomics Research Centre, DNAFit Ltd, London, UK

${ }^{5}$ Department of Health Sciences, Lehman College, Bronx, USA

${ }^{6} S$ chool of Medical and Health Sciences, Edith Cowan University, Joondalup, Australia

\section{Correspondence to}

Jozo Grgic, Institute for Health and Sport (IHES), Victoria University, Melbourne, Australia; jozo.grgic@live.vu.edu.au

Accepted 10 March 2019 Published Online First 29 March 2019

\author{
ABSTRACT \\ Objective To systematically review, summarise and \\ appraise findings of published meta-analyses that \\ examined the effects of caffeine on exercise performance. \\ Design Umbrella review. \\ Data sources Twelve databases. \\ Eligibility criteria for selecting studies Meta-
} analyses that examined the effects of caffeine ingestion on exercise performance.

Results Eleven reviews (with a total of 21 metaanalyses) were included, all being of moderate or high methodological quality (assessed using the Assessing the Methodological Quality of Systematic Reviews 2 checklist). In the meta-analyses, caffeine was ergogenic for aerobic endurance, muscle strength, muscle endurance, power, jumping performance and exercise speed. However, not all analyses provided a definite direction for the effect of caffeine when considering the $95 \%$ prediction interval. Using the Grading of Recommendations Assessment, Development and Evaluation criteria the quality of evidence was generally categorised as moderate (with some low to very low quality of evidence). Most individual studies included in the published meta-analyses were conducted among young men.

Summary/conclusion Synthesis of the currently available meta-analyses suggest that caffeine ingestion improves exercise performance in a broad range of exercise tasks. Ergogenic effects of caffeine on muscle endurance, muscle strength, anaerobic power and aerobic endurance were substantiated by moderate quality of evidence coming from moderate-to-high quality systematic reviews. For other outcomes, we found moderate quality reviews that presented evidence of very low or low quality. It seems that the magnitude of the effect of caffeine is generally greater for aerobic as compared with anaerobic exercise. More primary studies should be conducted among women, middle-aged and older adults to improve the generalisability of these findings.

\section{INTRODUCTION}

In 2018, the IOC published a consensus statement regarding the effects of dietary supplements on exercise performance of athletes. ${ }^{1}$ The consensus statement placed meta-analyses at the top of the evidence pyramid. ${ }^{1}$ In sports nutrition research, meta-analyses provide a method of pooling available primary studies exploring the efficacy of a given supplement on a specific outcome (eg, performance of an exercise test). As such, meta-analyses are used to support establishing evidence-based guidelines and decision making for the effective prescription of nutritional supplements and ergogenic aids.

One supplement with a long history of use for its ergogenic effects on performance is caffeine. ${ }^{2}$ Caffeine ingestion is highly prevalent among athletes, especially since 2004, when it was removed from the World Anti-Doing Agency list of within-competition banned substances. ${ }^{3}$ For example, $74 \%$ of urine samples collected from 2004 to 2008 and analysed as a part of doping control contained caffeine. ${ }^{3}$ Given inconsistent evidence in the primary research that examined the effects of caffeine on exercise performance, several research groups explored this area using metaanalytical methods. ${ }^{4-15}$ While these meta-analyses generally report ergogenic effects of caffeine on exercise performance, even adequately conducted meta-analyses tend to focus on the ergogenic effects of caffeine within just a single performance domain. As an illustration, Grgic and Pickering ${ }^{10}$ only examined the effects of caffeine ingestion on isokinetic peak torque.

Given that each meta-analysis is typically focused only on a specific aspect of exercise performance, it is challenging to: (1) compare the effects of caffeine ingestion on different performance domains; (2) comparatively assess the availability and strength of evidence for different performance domains; (3) establish comprehensive recommendations on the use of caffeine in sports and exercise; and (4) provide overall recommendations for future research on the ergogenic effects of caffeine on exercise performance. Such recommendations may increase the uptake of evidence-based findings in the context of supplement prescription and guide future research in this area.

Consistency of meta-analytical findings is often lacking; even meta-analyses that have examined the same outcome may produce conflicting findings. For instance, Gonçalves Ribeiro et $a l^{8}$ did not observe significant effects of caffeine ingestion on power. In contrast, a subsequent meta-analysis by Grgic $^{9}$ reported that caffeine ingestion is ergogenic for this outcome. Such conflicting findings hinder firm evidence-based conclusions from individual meta-analyses. Ultimately, the methods employed in a specific meta-analysis (eg, the number of databases searched, the comprehensiveness of the search syntax and the methods used for analysing the data) determine the robustness of the pooled results. For example, a meta-analysis on the effects of caffeine 
supplementation on power conducted by Gonçalves Ribeiro et $a l^{8}$ included only studies that were published between January 2010 and December 2015. Due to these restrictions, studies published before 2010 were excluded from consideration, and the authors provided no rationale for their approach. Only four studies that assessed power during Wingate tests were included in their review, and no significant pooled effects were found. Grgic $^{9}$ conducted a similar meta-analysis without any restrictions regarding the year of publication; this analysis included 16 studies and reported significant improvements in both mean and peak power on the Wingate test following caffeine ingestion.

One proposed method to overcome some of the above, and other, potential limitations of meta-analyses is to perform umbrella reviews. ${ }^{16}$ Umbrella reviews (ie, reviews that include the syntheses and appraisal of existing systematic reviews and meta-analyses) provide a comprehensive view of the evidence landscape on a given topic because they encompass larger scale of evidence. ${ }^{16}$ Such reviews help us to understand the current strengths and limitations of the entire body of evidence by comparing and contrasting findings from the entirety of the published data. Such a treatise on the effects of caffeine on exercise may be a useful resource for researchers, sports nutritionists, athletes, coaches and others interested in the ergogenic effects of caffeine on acute exercise performance. To date, there are no published umbrella reviews focusing on the effects of caffeine on exercise performance.

The aim of the present paper is threefold: (1) to systematically review available meta-analytical evidence that has examined the effects of caffeine on exercise performance; (2) to addresses the quality, strengths and limitations of the meta-analytical evidence; and (3) to identify current gaps in the literature and make key suggestions for future research.

\section{METHODS}

\section{Search strategy}

This review followed the guidelines set forth by Aromataris and colleagues. ${ }^{16}$ We systematically searched through 12 different databases, including: Academic Search Premier, AUSPORT, CINAHL, Cochrane Library, ERIC, Health Source: Nursing/ Academic Edition, MasterFILE Premier, PsycINFO, PubMed/ MEDLINE, Scopus, SPORTDiscus and Web of Science. The databases were searched from the inception of indexing until 24 September 2018 using the following search syntax: caffeine AND (meta-an* OR 'systematic review') AND (exercise OR training OR muscle OR 'physical performance'). The search syntax for each database is provided in online supplementary table S1. Quotation marks and the wildcard symbol were used to narrow down the search. In each full-text that was read, we also screened the reference list as a part of a secondary search. The search was carried out independently by two authors (JG and IG) to prevent any selection bias. The authors independently examined the titles, abstracts and, when applicable, the full-texts of the identified publications. On examination, the authors compared their lists of included and excluded papers; any disagreements were resolved by discussion and agreement between the authors.

\section{Inclusion criteria}

We included reviews coupled with a meta-analysis that examined the acute effects of caffeine ingestion on any exercise performance-related outcome. Both peer-reviewed and conference papers published in English or other languages were considered. Meta-analyses that included studies that combined caffeine with other ergogenic compounds, such as taurine, were excluded as they do not allow for the differentiation of the effects between the compounds. However, meta-analyses that included studies comparing caffeine and carbohydrate ingestion versus caffeine alone were included as long as the effect of caffeine could be isolated (ie, two solutions were given to the participants-one with caffeine and one without). As reported by the ParticipantIntervention-Comparison-Outcome (PICO) process, the following criteria were followed:

\section{Participants}

Apparently healthy individuals of both sexes and all ages.

\section{Interventions}

Any acute study examining the effects of caffeine ingestion on exercise performance.

\section{Comparison group}

Placebo (provided that the effects of caffeine could be isolated).

\section{Outcome measures}

Any form of exercise performance.

\section{Data extraction}

The following data were extracted from the included metaanalyses: (1) the list of authors and year of publication; (2) the number and type of studies included in the meta-analysis; (3) the pooled number of participants; (4) the type of exercise test that was evaluated; (5) the pooled effect size with the 95\% CI; (6) $\mathrm{p}$ values; and (7) percent changes and $\mathrm{I}^{2}$ values. The same two authors that carried out searches also conducted the data extraction process. All data were tabulated to a spreadsheet predefined for this review. After data extraction, the spreadsheets were cross-checked between the authors for accuracy.

\section{Methodological quality evaluation}

The methodological quality of the included meta-analyses was assessed using the validated Assessing the Methodological Quality of Systematic Reviews 2 (AMSTAR 2) checklist. ${ }^{17}$ Two reviewers (JG and IG) independently assessed the methodological quality of the included reviews using the AMSTAR 2 checklist. This checklist contains 16 items that include questions regarding the use of the PICO description as a part of the inclusion criteria, the a priori registration of the review design, the comprehensiveness of the literature search, the number of authors that performed that search and data extraction, the description of included studies, the assessment of the quality of the included primary studies, reporting of sources of funding in the primary studies, the use of appropriate statistical methods, assessments of heterogeneity in the meta-analyses and reporting of the potential conflicts of interest. Full details on the checklist can be found in the paper by Shea et al. ${ }^{17}$ Each item on this checklist is answered with a 'yes', 'no', 'cannot answer' or 'not applicable'. Out of these possible answers, only the 'yes' answer counts as a point in the total score for the assessed review. Based on the summary point scores, the meta-analyses were categorised as high quality (at least $80 \%$ of the items were satisfied), moderate quality (between $40 \%$ and $80 \%$ of the items were satisfied) or low quality (less than $40 \%$ of the items were satisfied), as performed previously. ${ }^{18} 19$ 


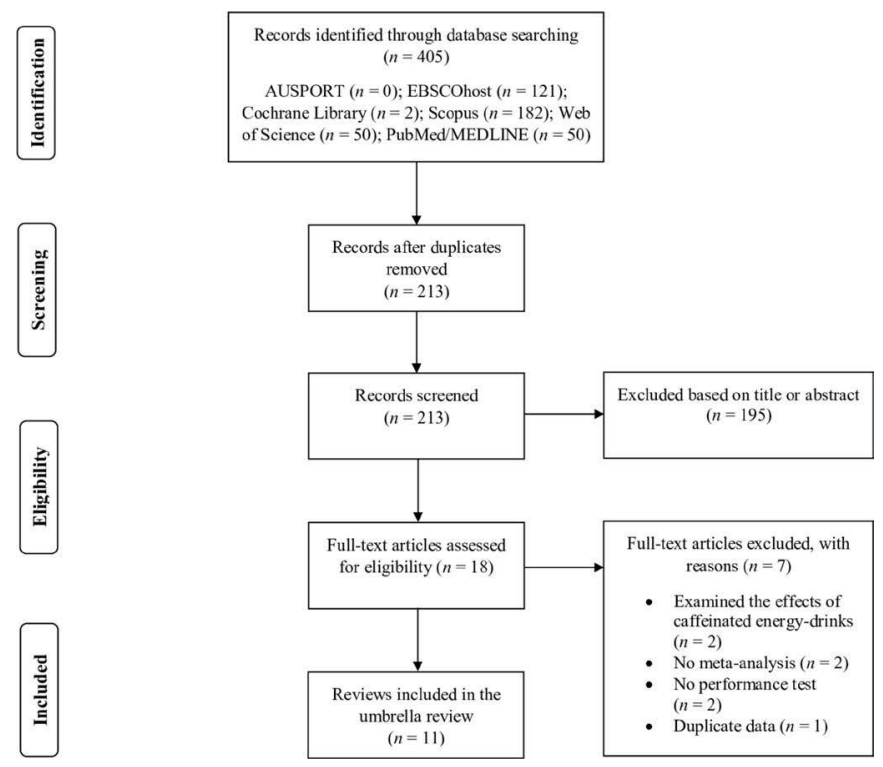

Figure 1 Flow diagram of the search process.

\section{Quality of evidence}

To assess the quality of evidence we used the modified Grading of Recommendations Assessment, Development and Evaluation (GRADE) principles. ${ }^{20}$ For the purpose of this review, we examined the following GRADE aspects: (1) risk of bias (determined by the quality of the primary studies, as assessed in the original reviews); (2) inconsistency (determined by variables such as the variation in the effects across the included studies and the overlap of the 95\% CIs between the studies); (3) indirectness (determined by the generalisability of the findings while considering the study populations included in the primary research); (4) imprecision (determined by the total sample size in the analysis and the width of the $95 \% \mathrm{CI}$ of the pooled effect size); and (5) publication bias (determined if the effect size of the largest study in each analysis was smaller than the pooled estimate from the meta-analysis and by examining the asymmetry of the funnel plot). Based on these criteria, the meta-analytical evidence was classified as high, moderate, low or very low. The GRADE assessment was conducted independently by two authors (JG and IG), with discussion and agreement for any differences.

\section{Prediction interval $(\mathrm{PI})$}

Using the number of included studies, the pooled standardised mean difference, the upper limit of the 95\% CI and the tausquared values (from each analysis), we calculated 95\% PI for all included meta-analyses (spreadsheet available at: https:// www.meta-analysis.com/pages/prediction.php). The 95\% PI represents the range in which the effect size of a future study conducted on the topic will most likely lie. If the tau-squared values were not provided in the meta-analysis, these data were either requested from the authors or recalculated based on the data presented in the included studies.

\section{RESULTS}

\section{Search results}

The initial literature search identified 405 search records. Out of that pool of search results, 18 full-texts were read. Seven reviews were excluded after reading the full-texts. ${ }^{721-26}$ The reasons for their exclusion are provided in online supplementary table S2. Eleven reviews (with a total of 21 meta-analyses) were included in this umbrella review. ${ }^{4-6}$ 8-15 All included reviews were published in peer-reviewed journals. The flow diagram of the search process can be found in figure 1 .

\section{Characteristics of the meta-analyses}

The included meta-analyses were published between the years 2004 and 2018. The median number of studies included per meta-analysis was 19 (range: 2-44). The prevalence of primary studies with male-only samples ranged from $72 \%$ to $100 \%$ across the meta-analyses. The assessed outcomes in the metaanalyses included: maximal speed during running, cycling or rowing (defined as the maximal achieved speed in exercise performance tests lasting from $45 \mathrm{~s}$ to $8 \mathrm{~min}$ that had either a fixed duration or a fixed distance), aerobic endurance (assessed by time-to-exhaustion, time trial and graded exercise tests), peak and mean power in the $30 \mathrm{~s}$ Wingate test, peak torque in an isokinetic strength assessment, strength in the one repetition maximum (1RM) test, height in a vertical jump test, muscular endurance (assessed both using isometric and dynamic tests), duration of time trial or power during a time trial and maximal voluntary strength (assessed by pooling isometric, isokinetic and $1 \mathrm{RM}$ tests). A summary of the included meta-analyses can be found in table 1 .

\section{Effects of caffeine on exercise performance}

The effects of caffeine ingestion on aerobic endurance were examined in five reviews with a total of nine meta-analyses; the majority reported ergogenic effects of caffeine (effect size range: $0.22-0.61)$. The range of included primary studies was from 2 to 44 (average: 23 studies). Doherty and Smith ${ }^{6}$ did not report significant effects of caffeine on aerobic endurance performance when considering only graded exercise tests and including six studies. Gonçalves Ribeiro $e \mathrm{al}^{8}$ did not report significant effects of caffeine on this outcome (analysed using maximum running distance tests) while including two studies. The 95\% PIs for these analyses are reported in table 1.

Four analyses examined the effects of caffeine on different measures of muscle strength. In three of these analyses, an ergogenic effect of caffeine was observed (effect size range: 0.16 0.20 ). The range of included studies was from 3 to 27 (average: 13 studies). In the analysis by Grgic and Pickering, ${ }^{10}$ the $95 \%$ PI was from -0.17 to 0.49 . In the analysis by Grgic et al, ${ }^{11}$ the 95\% PI was from 0.02 to 0.39 , while in Warren et al's ${ }^{15}$ analysis, the $95 \%$ PI was from -0.18 to 0.56 . The $95 \%$ PI in the analysis by Polito $e{ }^{a} l^{12}$ (this analysis did not report significant effects of caffeine on 1RM strength) was from -0.09 to 0.27 .

Two analyses examined the effects of caffeine on muscular endurance. Both reported ergogenic effects of caffeine (effect size range: $0.28-0.38$ ). Polito et al ${ }^{12}$ included 16 , while Warren et $a l^{15}$ included 23 studies. The 95\% PI was from 0.02 to 0.74 and from -0.29 to 0.85 for the analyses by Polito et $a l^{12}$ and Warren et al, ${ }^{15}$ respectively.

Anaerobic power was examined in three analyses. In a metaanalysis including four studies, Gonçalves Ribeiro et $a l^{8}$ did not report significant ergogenic effects of caffeine on power. The $95 \%$ PI in this analysis was from -0.65 to 1.01 . In an analysis including 16 studies, Grgic ${ }^{9}$ reported ergogenic effects of caffeine on both mean and peak power (effect size range: 0.18 to 0.27 ). In the analysis for peak power, the 95\% PI was from -0.35 to 0.89 , while in the analysis for mean power, the $95 \%$ PI was from 0.04 to 0.32 .

One meta-analysis, including 10 studies, examined the effects of caffeine on vertical jump height and reported an ergogenic 


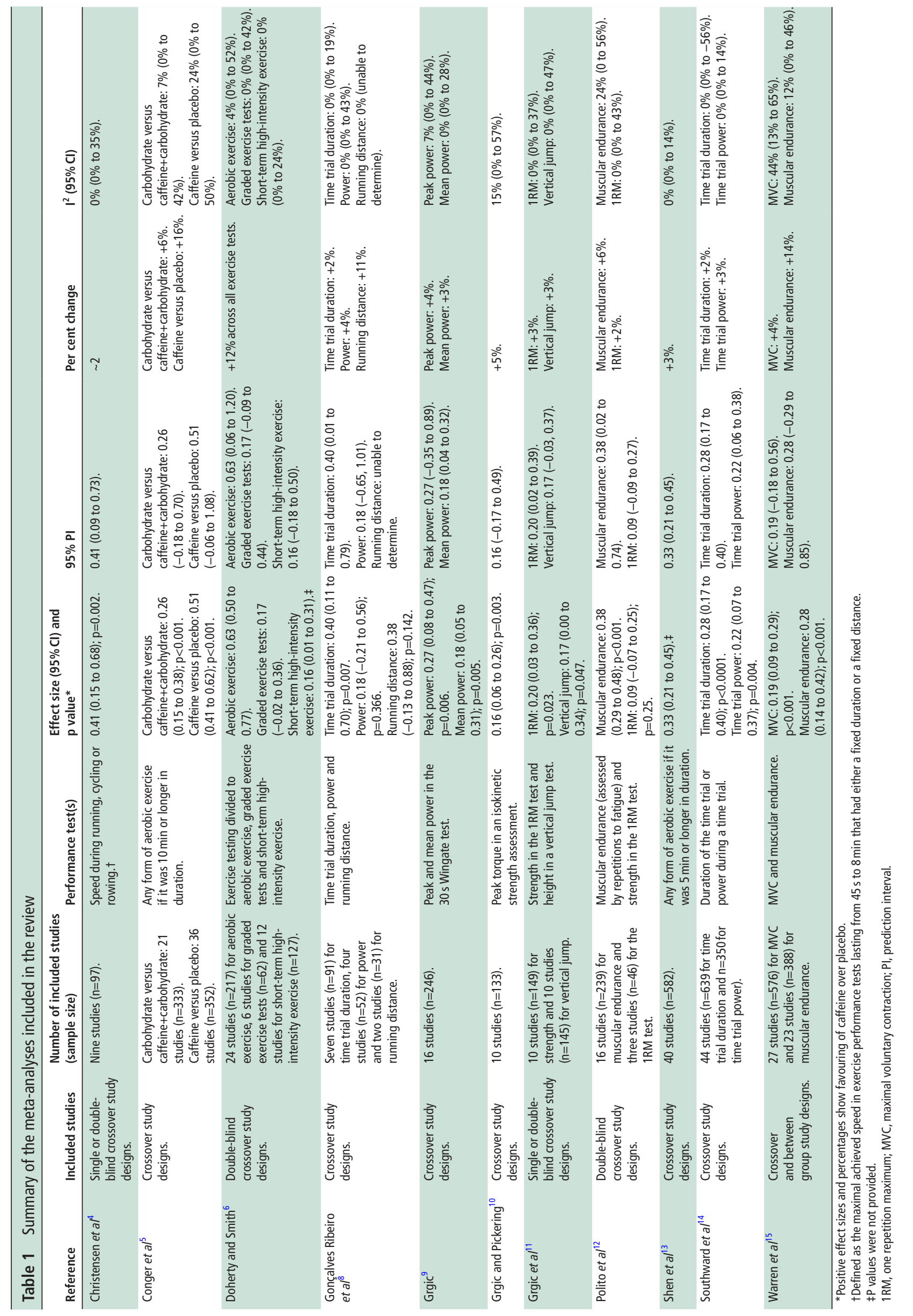




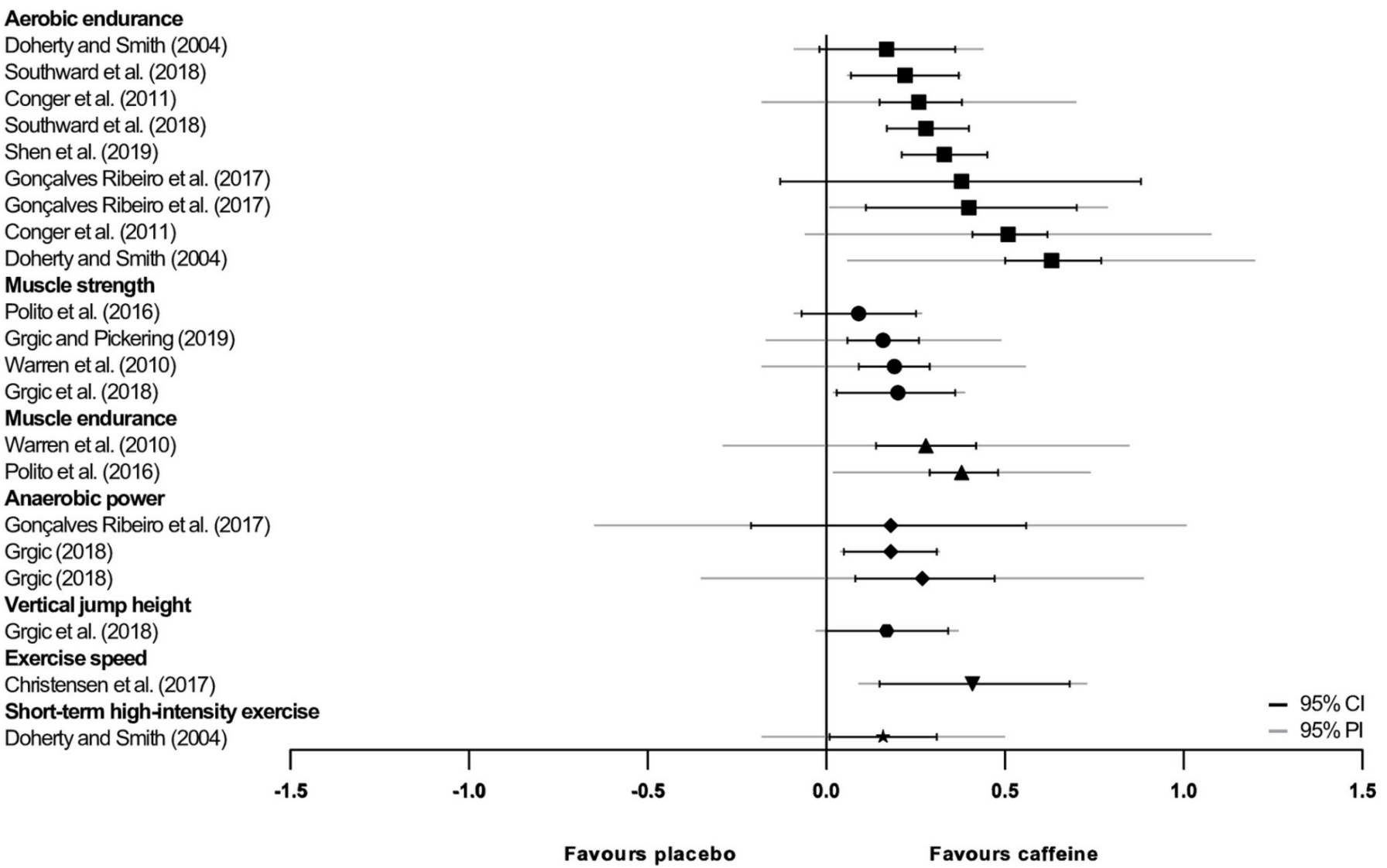

Figure 2 Summary of the effect sizes, 95\% Cls (presented in the black lines), and 95\% prediction intervals (95\% PIs; presented in the grey lines) from the included meta-analyses. If there is no $95 \% \mathrm{PI}$ presented, it was the same as the $95 \% \mathrm{Cl}$.

effect of caffeine (effect size: 0.17). ${ }^{11}$ The 95\% PI was from -0.03 to 0.37 .

One meta-analysis, included nine studies, examined speed during running, cycling or rowing and reported ergogenic effects of caffeine (effect size: 0.41 ). ${ }^{4}$ The $95 \%$ PI was from 0.09 to 0.73 .

One meta-analysis examined various forms of 'short-term high-intensity exercise' while pooling the effects of caffeine on: (1) time to exhaustion in various high-intensity short-term cycling and running efforts; (2) mean power, peak power output and total work during high-intensity short-term cycling; and (3) time trial time during $2000 \mathrm{~m}$ rowing. ${ }^{6}$ This analysis included 16 studies and reported ergogenic effects of caffeine of 0.16 ; the $95 \%$ PI was -0.18 to 0.50 .

Besides the main analysis (presented in figure 2), several reviews also conducted additional subgroup analyses (eg, for trained vs untrained individuals, for upper vs lower body musculature) and these results are summarised in online supplementary table S3.

Methodological quality evaluation

The methodological quality of the 11 included reviews is summarised in table 2 . The reviews scored from $44 \%$ to $88 \%$ of the maximum 16 points. Three reviews were classified as being of high quality, while eight were classified as being of moderate methodological quality. None of the reviews were considered to be of poor methodological quality. Several criteria on AMSTAR 2 checklist were under-reported in the analysed reviews: (1) none provided an a priori design (ie, registration of the review methods in advance); (2) in four and five analyses the number of authors conducting the search and data extraction was not clear, respectively; (3) the list of excluded studies was not provided in any of the included reviews; and (4) sources of funding for the studies included in a given review were discussed only in three reviews.

\section{Quality of the evidence}

Based on the GRADE assessment, the included analyses were considered as providing very low (3 meta-analyses), low (7 metaanalyses) or moderate quality of evidence (11 meta-analyses). For risk of bias, several reviews did not assess the quality of the included studies and thus were given 'unclear' on this criterion. The meta-analyses were considered as not having serious inconsistency but were considered as having serious indirectness. The analyses were mostly considered as being 'precise' on the imprecision GRADE item. Finally, three meta-analyses were considered as 'strongly suspected' on the publication bias GRADE item. The results for each analysis are presented in online supplementary table S4.

\section{DISCUSSION}

Based on the 11 included reviews, it can be concluded that caffeine is ergogenic for different components of exercise performance including aerobic endurance, muscle strength, muscle endurance, power, jumping performance and exercise speed. Ergogenic effects of caffeine on muscle endurance, muscle strength, anaerobic power and aerobic endurance were substantiated by moderate quality of evidence coming from moderateto-high quality systematic reviews (online supplementary table 


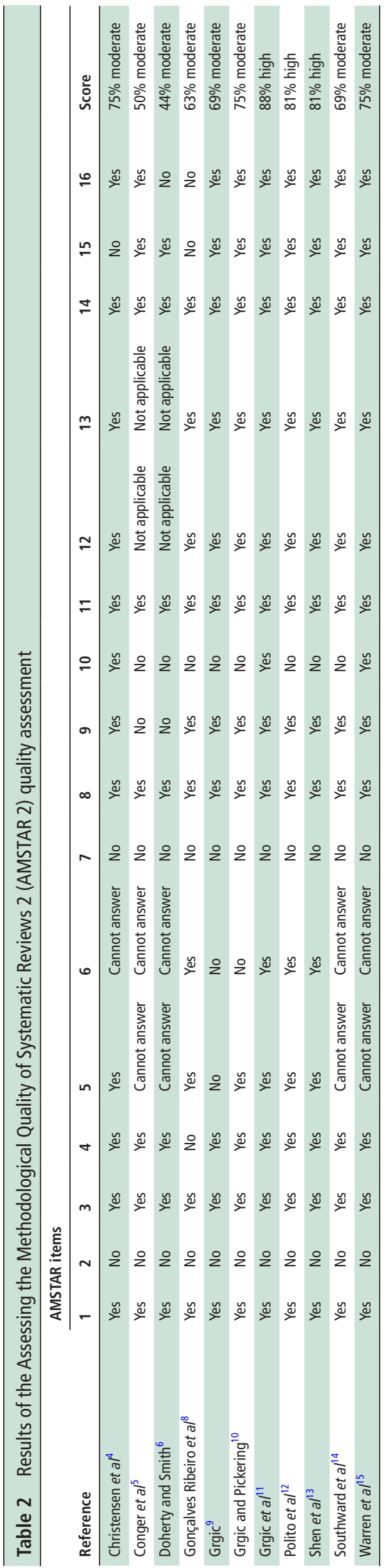

S5). For other outcomes, we found moderate quality reviews that presented evidence of very low or low quality. In addition, not all analyses provided a definite direction for the effect of caffeine when considering the 95\% PI. Several important aspects that refer to the generalisability of the meta-analytical findings as well as the spread of summary effects need to be considered when interpreting these findings from a practical standpoint.

\section{Generalisability of the results}

Based on the GRADE assessment for directness of evidence, the included reviews were rated as having serious indirectness given that the evidence is not direct enough to apply to all populations. Scrutiny of the meta-analyses included in this umbrella review highlights that primary studies conducted among women are lacking. Specifically, in all of the included meta-analyses, $72 \%-100 \%$ of the pooled sample participants were men. Women may metabolise caffeine differently than men given that changes in circulating steroid hormones during phases of the menstrual cycle can impact caffeine elimination in women, ${ }^{2728}$ which might also impact the ergogenic effect of caffeine on exercise performance in this population. When conducting studies in women, the differences in caffeine metabolism across the follicular and luteal phase of the menstrual cycle may increase the complexity of the study design, which might partially explain why studies in this population are lacking. While there are studies conducted in both sexes that suggest both men and women may experience similar acute effects of caffeine ingestion on exercise performance ${ }^{29}{ }^{30}$ the generalisability of the meta-analytic findings is, however, limited mostly to men.

The majority of the primary studies were conducted in young individuals, and therefore, several meta-analyses are limited exclusively to young individuals. ${ }^{48911-14}$ This may be relevant to highlight given that in animal models, with ageing, there appears to be a reduced ergogenic effect of caffeine. ${ }^{31}$ Caffeine has been shown to elicit positive effects on mood and cognitive performance in older adults. ${ }^{32}$ If caffeine also increases exercise performance in older adults, it might also enhance performance during activities of daily living in these individuals. This is particularly important from a public health point of view, given that reduced physical functioning (eg, in terms of reduced strength) may impact the quality of life in this population group. ${ }^{33}$ Although some of the studies conducted in older adults showed an ergogenic effect of caffeine on exercise performance, ${ }^{3435}$ additional studies that directly compare the effects of caffeine between young versus older individuals are needed to explore if the effects of caffeine differ between age groups.

\section{Methodological quality}

While the meta-analyses included in the present umbrella review show that caffeine ingestion may indeed be ergogenic across a large range of exercise tasks, some additional considerations may help to improve future meta-analyses on this topic. Several of the included reviews did not adhere to the Preferred Reporting Items for Systematic Reviews and Meta-Analyses (PRISMA) guidelines, which currently represent a widely accepted standard for reporting meta-analyses. It should be taken into account that the PRISMA guidelines were published in 2009, which is 5 years after the review by Doherty and Smith. ${ }^{6}$ Nonetheless, several meta-analyses that did not follow the guidelines were published following the release of the PRISMA statement. ${ }^{45815}$

None of the 11 reviewes registered their protocol for a review and thus did not receive a point on item 2 of the AMSTAR 2 checklist. Protocols of systematic reviews can be registered in 
the PROSPERO database. If registered, such protocols can help reduce the risk of wasteful duplication of reviews by independent research groups. However, the PROSPERO database is primarily focused on health outcomes and not exercise performance. As stated on their website, 'PROSPERO includes protocol details for systematic reviews relevant to health and social care, welfare, public health, education, crime, justice, and international development, where there is a health related outcome'. The authors are not aware of any registries that focus on the publishing of protocols for systematic reviews in the sport and exercise field. Given that the number of published systematic reviews has increased over the last years, the formation of such a register for this line of research appears warranted.

Publication bias, as highlighted by Borenstein et al, ${ }^{36}$ can occur because studies that report higher (and significant) effect sizes are more likely to be published than those with low or non-significant effect sizes (ie, the file drawer problem). Therefore, the inclusion of only published studies in a given metaanalysis can lead to publication bias and may be a concern for the validity of the results. Four meta-analyses included in this umbrella review also examined unpublished literature in the form of master's theses and doctoral dissertations. ${ }^{511} 1215$ In the meta-analysis by Conger et $a l,{ }^{5}$ the effect size of the unpublished studies was 0.13 ( $95 \% \mathrm{CI}-0.08$ to 0.33 ), while the effect size of the published studies was 0.32 (95\% CI 0.19 to 0.46 ). These results might indeed suggest that studies with smaller effect sizes tend to remain unpublished and to avoid publication bias future meta-analyses should consider including unpublished results as well. The reviews that included unpublished literature highlight that, in many cases, such unpublished documents may be of equal or even greater methodological quality as those found in peer-reviewed journals. The influence of unpublished results can be examined by conducting a sensitivity analysis in which the pooled results are inspected after the exclusion of these studies. In this context, journal editors and reviewers are also encouraged to facilitate greater acceptance and publication of studies with results that would appear to be 'less favourable' (or statistically non-significant) to truly progress this area of work.

\section{The spread of summary effects}

Based on the GRADE assessment of inconsistency, the reviews were classified as not possessing serious inconsistencies. Indeed, the effect sizes across individual studies indicate that the studies rarely show a negative effect of caffeine supplementation on exercise performance. The effects in the primary studies were either positive or around the null value. In addition, the $95 \% \mathrm{CI}$ from the primary studies largely overlap.

One interesting aspect refers to the spread of summary effects. Historically, caffeine ingestion has been suggested to predominantly provide a performance-enhancing effect on aerobic exercise performance. ${ }^{37}$ As shown both here and by others, ${ }^{38}{ }^{39}$ it is evident that caffeine ingestion enhances performance in anaerobic exercise tasks as well. However, it is possible that the magnitude of the effect of caffeine is greater for aerobic as compared with anaerobic exercise. The effect sizes for meta-analyses that focused on aerobic tests of performance are generally higher than those that used anaerobic tests of performance (figure 2). Future studies may consider investigating the effects of caffeine ingestion on both aerobic and anaerobic tests of performance in the same sample to further explore whether the effect size magnitude differs between tasks that rely on predominantly oxidative or predominantly non-oxidative energy pathways.
The optimal dose of caffeine

While the included meta-analyses report that caffeine ingestion may be ergogenic across a broad range of exercise activities, the 'optimal' dose of caffeine remains elusive. If we observe the dosage used in the primary studies (across all of the included meta-analyses), it is clear that most of the studies used a single dose of caffeine (most commonly $6 \mathrm{mg} / \mathrm{kg}$ ). Warren $e^{\mathrm{a}} \mathrm{al}^{15}$ examined the dose-response effects between the amount of caffeine ingested and its ergogenic effect on muscular endurance. This analysis found that for an increase in caffeine dose by $1 \mathrm{mg} /$ $\mathrm{kg}$, the relative effect size for muscular endurance increased by 0.10 . However, these results should be interpreted with caution given that the dosage explained only $16 \%$ of the between-study variance. To explore the optimal doses of caffeine for exercise performance future dose-response studies are needed. The optimal doses may differ based on the source of caffeine ${ }^{40}$ exercise test, ${ }^{41-44}$ muscle action type ${ }^{45}$ and between individuals, ${ }^{46} 47$ which needs to be taken into account when prescribing caffeine supplementation.

\section{Is coffee a good way to consume caffeine?}

While the results of this umbrella review suggest that caffeine is ergogenic in the majority of exercise situations, it is important to keep in mind that the majority of studies use caffeine anhydrous (highly concentrated caffeine powder) as the caffeine source, with a smaller group of studies utilising caffeine-containing supplements such as energy drinks, bars and gels. Coffee-the most widely used method of caffeine ingestion globally-is relatively underexplored as a pre-exercise performance enhancer. Hodgson and colleagues ${ }^{48}$ reported that caffeine anhydrous and coffee, standardised to deliver a caffeine dose of $5 \mathrm{mg} / \mathrm{kg}$, were similarly effective in enhancing aerobic endurance performance. Similar results have been reported for resistance and sprint exercise. ${ }^{4950}$ As a result, coffee is likely an effective ergogenic aid; the main issue here is a practical one. To be ergogenic, the caffeine dose from coffee likely has to fall within the $3-6 \mathrm{mg} / \mathrm{kg}$ range. The caffeine dose received from coffee depends on many factors, including bean type, preparation method and size of the cup; there are large differences in caffeine concentrations between different coffee brands and flavours and within the same brand across time ${ }^{51-53}$ As a result, while the 'average' cup of coffee contains around $100 \mathrm{mg}$ of caffeine-meaning that two cups would deliver $\sim 200 \mathrm{mg}$, representing $\sim 3 \mathrm{mg} / \mathrm{kg}$ for a $70 \mathrm{~kg}$ individual-this amount is hard to quantify in the specific cup of coffee that person is drinking. ${ }^{51}$ While keeping those caveats in mind, as a broad rule of thumb, two cups of coffee, consumed around $60 \mathrm{~min}$ before exercise, should exert an ergogenic effect in most individuals.

\section{Suggestions for future research}

Subgroup analyses conducted in the included meta-analyses in most cases are based on a low number of included studies (or effect sizes), which limits any definitive conclusions. Many areas remain unclear when it comes to caffeine supplementation. Some of these areas include:

\section{The effects of caffeine habituation}

Does habituation to caffeine reduce (or eliminate) its ergogenic effect following acute caffeine supplementation? The included meta-analyses could not explore the differences in effects between low and high habitual caffeine users as currently there is a lack of primary studies investigating this topic. The body of research is limited and equivocal, with some studies suggesting 
that low habitual caffeine users experience greater ergogenic effects than the high habitual users, while others report similar acute responses to caffeine ingestion in terms of exercise performance regardless of habituation. ${ }^{54}$ Pickering and Kiely ${ }^{56}$ suggested the possibility that the response may be dose dependent, which may be an interesting aspect to explore in future studies.

\section{Optimal timing of caffeine ingestion}

Most studies provided caffeine supplementation $60 \mathrm{~min}$ pre exercise; therefore, it remains unclear if smaller/greater effects of caffeine would be observed with shorter/longer wait time from ingestion to exercise. This area needs further exploration, and there is potential that different timing may be required for different doses ${ }^{57}$ or genotypes. ${ }^{58}$

\section{Effects of different sources of caffeine}

Most of the included studies in the meta-analyses used the capsule form of caffeine. It remains unclear if comparable results can also be seen with alternate sources of caffeine, such as caffeine mouth rinsing, caffeine gels and chewing gums. A detailed review on the topic of alternate forms of caffeine can be found elsewhere. ${ }^{40}$

Effects of caffeine among trained versus untrained individuals

While it has been suggested that trained individuals might respond better to caffeine ingestion, the current evidence on this topic is scarce and conflicting. ${ }^{59-61}$ The meta-analyses that have tried to explore this matter were commonly performed on a limited number of studies. For example, Grgic et al ${ }^{11}$ only included seven and four studies for their subgroup analysis of the effects of caffeine among trained and untrained individuals, respectively. The majority of the studies pooled in the mentioned subgroup analysis only examined the effects of caffeine on strength performance in either trained or untrained individuals. The only study included in the review by Grgic et $a l^{11}$ that directly compared the effects of caffeine between trained and untrained individuals reported ergogenic effects of caffeine in untrained but not in trained individuals. ${ }^{59}$ These results are in contrast to the common belief about greater responsiveness to caffeine in trained individuals. Future work is needed on this topic (for additional discussion on this topic see the reviews by Tallis et $a l^{62}$ and Burke ${ }^{63}$ ).

\section{Chronic effects of caffeine on exercise adaptations}

While many studies have examined the acute effects of caffeine supplementation on exercise performance, it remains unclear whether these acute increases in performance also impact chronic adaptations to training and in which way. Ultimately, individuals interested in the acute performanceenhancing effects of caffeine are likely candidates to continue to use caffeine supplementation over the long term. Aspects of long-term supplementation that refer to habituation and to the attenuation of caffeine's effects, as well as the effects of chronic caffeine supplementation on training adaptations, need to be further investigated.

We hope that highlighting some of these areas will help catalyse future high-quality research.

\section{CONCLUSIONS}

Caffeine ingestion may be ergogenic for a broad range of exercise tasks. The performance-enhancing effects of caffeine on:
What is already known

- Given the often narrow scope (ie, focus on only one test of performance) of a meta-analysis, the credibility of this type of evidence for the effects of caffeine on exercise performance across the totality of the evidence is unclear.

- Caffeine is ergogenic for exercise performance; it remains unclear if the effect of caffeine differs between various exercise tests/tasks.

\section{What are the new findings}

- Of the 11 included reviews, all report significant improvements in at least one component of exercise performance following caffeine ingestion with the effect size magnitude ranging from small to moderate.

- The effect sizes for meta-analyses that focused on aerobic tests of performance are generally higher than those that used anaerobic tests of performance.

- The meta-analytic findings apply mostly to men and young individuals.

- Caffeine ingestion (as studied here) does not translate readily to 'coffee' - the drink, but begs an obvious question. As a broad rule of thumb, two cups of coffee, consumed around $60 \mathrm{~min}$ before exercise, should exert an ergogenic effect in most individuals.

(A) muscle endurance, (B) muscle strength, (C) anaerobic power and (D) aerobic endurance were supported by moderate-to-high quality reviews and moderate quality of evidence. For other outcomes, even though the reviews were of moderate quality, the evidence was of very low or low quality. The magnitude of the effect of caffeine is generally greater for aerobic as compared with anaerobic exercise.

Contributors JG and ZP conceived the idea for thereview. JG and IG conducted the study selection the data extraction and qualityassessment. ZP contributed to data extraction and conceptualisation of qualityassessment. JG drafted the initial manuscript. CP, ZP, IG, BJS, and DJBcontributed to writing the manuscript.

Funding The authors have not declared a specific grant for this research from any funding agency in the public, commercial or not-for-profit sectors.

Competing interests None declared.

Patient consent for publication Not required.

Provenance and peer review Not commissioned; externally peer reviewed.

\section{ORCID iDs}

Jozo Grgic http://orcid.org/0000-0002-6929-2844

Craig Pickering http://orcid.org/0000-0002-7056-4966

Zeljko Pedisic http://orcid.org/0000-0003-2886-3556

\section{REFERENCES}

1 Maughan RJ, Burke LM, Dvorak J, et al. IOC consensus statement: dietary supplements and the high-performance athlete. Br J Sports Med 2018;52:439-55.

2 Rivers WH, Webber HN. The action of caffeine on the capacity for muscular work. J Physiol 1907;36:33-47.

3 Del Coso J, Muñoz G, Muñoz-Guerra J. Prevalence of caffeine use in elite athletes following its removal from the World Anti-Doping Agency list of banned substances. Appl Physiol Nutr Metab 2011;36:555-61.

4 Christensen PM, Shirai Y, Ritz C, et al. Caffeine and bicarbonate for speed. a metaanalysis of legal supplements potential for improving intense endurance exercise performance. Front Physiol 2017;8:240.

5 Conger SA, Warren GL, Hardy MA, et al. Does caffeine added to carbohydrate provide additional ergogenic benefit for endurance? Int I Sport Nutr Exerc Metab 2011;21:71-84. 
6 Doherty M, Smith PM. Effects of caffeine ingestion on exercise testing: a metaanalysis. Int J Sport Nutr Exerc Metab 2004;14:626-46.

7 Doherty M, Smith PM. Effects of caffeine ingestion on rating of perceived exertion during and after exercise: a meta-analysis. Scand I Med Sci Sports 2005; 15:69-78.

8 Gonçalves Ribeiro B, Pontes Morales A, Sampaio-Jorge F, et al. Acute effects of caffeine intake on athletic performance: a systematic review and meta-analysis. Rev Chil Nutr 2017:44:283-91.

9 Grgic J. Caffeine ingestion enhances Wingate performance: a meta-analysis. Eur J Sport Sci 2018:18:219-25.

10 Grgic J, Pickering C. The effects of caffeine ingestion on isokinetic muscular strength: A meta-analysis. J Sci Med Sport 2019;22:353-60.

11 Grgic J, Trexler ET, Lazinica B, et al. Effects of caffeine intake on muscle strength and power: a systematic review and meta-analysis. I Int Soc Sports Nutr 2018;15:11.

12 Polito MD, Souza DB, Casonatto J, et al. Acute effect of caffeine consumption on isotonic muscular strength and endurance: a systematic review and meta-analysis. $\mathrm{SC}$ Sports 2016:31:119-28.

13 Shen JG, Brooks MB, Cincotta J, et al. Establishing a relationship between the effect of caffeine and duration of endurance athletic time trial events: A systematic review and meta-analysis. J Sci Med Sport 2019;22:232-8.

14 Southward K, Rutherfurd-Markwick KJ, Ali A. Correction to: the effect of acute caffeine ingestion on endurance performance: a systematic review and meta-analysis. Sports Med 2018;48:2425-41

15 Warren GL, Park ND, Maresca RD, et al. Effect of caffeine ingestion on muscular strength and endurance: a meta-analysis. Med Sci Sports Exerc 2010;42:1375-87.

16 Aromataris E, Fernandez R, Godfrey CM, et al. Summarizing systematic reviews: methodological development, conduct and reporting of an umbrella review approach Int J Evid Based Healthc 2015:13:132-40.

17 Shea BJ, Reeves BC, Wells G, et al. AMSTAR 2: a critical appraisal tool for systematic reviews that include randomised or non-randomised studies of healthcare interventions, or both. BMJ 2017;358:j4008.

18 Monasta L, Batty GD, Cattaneo A, et al. Early-life determinants of overweight and obesity: a review of systematic reviews. Obes Rev 2010;11:695-708

19 Johnson BT, MacDonald HV, Bruneau ML, et al. Methodological quality of meta-analyses on the blood pressure response to exercise: a review. J Hypertens 2014;32:706-23.

20 Guyatt G, Oxman AD, Akl EA, et al. GRADE guidelines: 1. IntroductionGRADE evidence profiles and summary of findings tables. I Clin Epidemiol 2011;64:383-94.

21 Brown SJ, Brown J, Foskett A. The effects of caffeine on repeated sprint performance in team sport athletes - a meta-analysis - Sport Sci Rev 2013:22:25-32.

22 Souza DB, Del Coso J, Casonatto J, et al. Acute effects of caffeine-containing energy drinks on physical performance: a systematic review and meta-analysis. Eur I Nutr 2017;56:13-27.

23 Astorino TA, Roberson DW. Efficacy of acute caffeine ingestion for short-term high-intensity exercise performance: a systematic review. I Strength Cond Res 2010;24:257-65.

24 Ganio MS, Klau JF, Casa DJ, et al. Effect of caffeine on sport-specific endurance performance: a systematic review. J Strength Cond Res 2009:23:315-24.

25 Glaister M, Gissane C. Caffeine and physiological responses to submaximal exercise: a meta-analysis. Int I Sports Physiol Perform 2018;13:402-11.

26 Zhang Y, Coca A, Casa DJ, et al. Caffeine and diuresis during rest and exercise: A meta-analysis. J Sci Med Sport 2015;18:569-74.

27 Lane JD, Steege JF, Rupp SL, et al. Menstrual cycle effects on caffeine elimination in the human female. Eur I Clin Pharmacol 1992;43:543-6.

28 Temple JL, Ziegler AM. Gender differences in subjective and physiological responses to caffeine and the role of steroid hormones. J Caffeine Res 2011;1:41-8.

29 Butts NK, Crowell D. Effect of caffeine ingestion on cardiorespiratory endurance in men and women. Res Q Exerc Sport 1985;56:301-5.

30 Sabblah S, Dixon D, Bottoms L. Sex differences on the acute effects of caffeine on maximal strength and muscular endurance. Comp Exerc Physiol 2015;11:89-94.

31 Tallis J, James RS, Cox VM, et al. Is the ergogenicity of caffeine affected by increasing age? The direct effect of a physiological concentration of caffeine on the power output of maximally stimulated EDL and diaphragm muscle isolated from the mouse. J Nutr Health Aging 2017:21:440-8.

32 Tallis J, Duncan MJ, Wright SL, et al. Assessment of the ergogenic effect of caffeine supplementation on mood, anticipation timing, and muscular strength in older adults. Physiol Rep 2013;1:e00072.

33 McPhee JS, French DP, Jackson D, et al. Physical activity in older age: perspectives for healthy ageing and frailty. Biogerontology 2016;17:567-80.
34 Norager $C B$, Jensen MB, Madsen MR, et al. Caffeine improves endurance in 75-yr-old citizens: a randomized, double-blind, placebo-controlled, crossover study. J App/ Physiol 2005;99:2302-6.

35 Duncan MJ, Clarke ND, Tallis J, et al. The effect of caffeine ingestion on functional performance in older adults. J Nutr Health Aging 2014;18:883-7.

36 Borenstein M, Hedges LV, Higgins JPT, et al. Introduction to meta-analysis. New York: Wiley, 2009:277-91.

37 Tarnopolsky MA. Caffeine and endurance performance. Sports Med 1994;18:109-25.

38 Davis JK, Green JM. Caffeine and anaerobic performance: ergogenic value and mechanisms of action. Sports Med 2009;39:813-32.

39 Grgic J, Mikulic P. Caffeine ingestion acutely enhances muscular strength and power but not muscular endurance in resistance-trained men. Eur J Sport Sci 2017:17:1029-36.

40 Wickham KA, Spriet LL. Administration of Caffeine in Alternate Forms. Sports Med 2018:48:79-91.

41 Pallarés JG, Fernández-Elías VE, Ortega JF, et al. Neuromuscular responses to incremental caffeine doses: performance and side effects. Med Sci Sports Exerc 2013;45:2184-92.

42 Sabol F, Grgic J, Mikulic P. The effects of three different doses of caffeine on jumping and throwing performance: a randomized, double-blind, crossover study. Int I Sports Physiol Perform 2019:1-25.

43 Grgic J, Mikulic P, Schoenfeld BJ, et al. The influence of caffeine supplementation on resistance exercise: a review. Sports Med 2019;49:17-30

44 Grgic J. Are there non-responders to the ergogenic effects of caffeine ingestion on exercise performance? Nutrients 2018;10:1736.

45 Tallis J, Yavuz HCM. The effects of low and moderate doses of caffeine supplementation on upper and lower body maximal voluntary concentric and eccentric muscle force. Appl Physiol Nutr Metab 2018:43:274-81.

46 Jenkins NT, Trilk JL, Singhal A, et al. Ergogenic effects of low doses of caffeine on cycling performance. Int I Sport Nutr Exerc Metab 2008;18:328-42.

47 Pickering C, Kiely J. Are the current guidelines on caffeine use in sport optimal for everyone? Inter-individual variation in caffeine ergogenicity, and a move towards personalised sports nutrition. Sports Med 2018:48:7-16.

48 Hodgson AB, Randell RK, Jeukendrup AE. The metabolic and performance effects of caffeine compared to coffee during endurance exercise. PLoS One 2013;8:e59561.

49 Trexler ET, Smith-Ryan AE, Roelofs EJ, et al. Effects of coffee and caffeine anhydrous on strength and sprint performance. Eur J Sport Sci 2016;16:702-10.

50 Richardson DL, Clarke ND. Effect of coffee and caffeine ingestion on resistance exercise performance. J Strength Cond Res 2016;30:2892-900.

51 Desbrow B, Hughes R, Leveritt M, et al. An examination of consumer exposure to caffeine from retail coffee outlets. Food Chem Toxicol 2007:45:1588-92.

52 Desbrow B, Henry M, Scheelings P. An examination of consumer exposure to caffeine from commercial coffee and coffee-flavoured milk. J Food Compos Anal 2012;28:114-8.

53 Desbrow B, Hall S, Irwin C. Caffeine content of Nespresso $\AA$ pod coffee. Nutr Health 2019;25:3-7.

54 Bell DG, McLellan TM. Exercise endurance 1, 3, and $6 \mathrm{~h}$ after caffeine ingestion in caffeine users and nonusers. J Appl Physiol 2002;93:1227-34.

55 Gonçalves LS, Painelli VS, Yamaguchi G, et al. Dispelling the myth that habitual caffeine consumption influences the performance response to acute caffeine supplementation. J Appl Physiol 2017;123:213-20.

56 Pickering C, Kiely J. What should we do about habitual caffeine use in athletes? Sports Med 2018.

57 Talanian JL, Spriet LL. Low and moderate doses of caffeine late in exercise improve performance in trained cyclists. Appl Physiol Nutr Metab 2016;41:850-5.

58 Pickering C. Caffeine, CYP1A2 genotype, and sports performance: is timing important? Ir J Med Sci 2019;188:349-50.

59 Brooks JH, Wyld K. Acute effects of caffeine on strength performance in trained and untrained individuals. J Athl Enhanc 2015;04:6.

60 Astorino TA, Cottrell T, Talhami Lozano A, et al. Effect of caffeine on RPE and perceptions of pain, arousal, and pleasure/displeasure during a cycling time trial in endurance trained and active men. Physiol Behav 2012;106:211-7.

61 Collomp K, Ahmaidi S, Chatard JC, et al. Benefits of caffeine ingestion on sprint performance in trained and untrained swimmers. Eur J Appl Physiol Occup Physiol 1992:64:377-80

62 Tallis J, Duncan MJ, James RS. What can isolated skeletal muscle experiments tell us about the effects of caffeine on exercise performance? Br J Pharmacol 2015;172:3703-13.

63 Burke LM. Caffeine and sports performance. Appl Physiol Nutr Metab 2008;33:1319-34. 